\title{
Transitive Nearest Neighbor Search in Mobile Environments *
}

\author{
Baihua Zheng $^{\dagger}$ Ken C.K. Lee ${ }^{\ddagger}$ Wang-Chien Lee ${ }^{\ddagger}$ \\ ${ }^{\dagger}$ Singapore Management University, Singapore. bhzheng@smu.edu.sg \\ ${ }_{\ddagger}^{\ddagger}$ Penn State University, PA16802, USA. \{cklee,wlee\}@cse.psu.edu
}

\begin{abstract}
Given a query point $p$, typically the position of a current client, and two datasets $\mathcal{S}$ and $\mathcal{R}$, a transitive nearest neighbor (TNN) search returns a pair of objects $(s, r) \in \mathcal{S} \times \mathcal{R}$ such that the total distance from $p$ to $s$ and then to $r$, i.e., dis $(p, s)+\operatorname{dis}(s, r)$, is minimum. We propose various algorithms for supporting TNN search as a kind of locationbased services in both on-demand-based and broadcastbased mobile environments. In addition, we develop a novel validation algorithm that allows the clients to verify whether their TNN query answers are still valid after they moved to new positions. Finally, we conduct a comprehensive simulation to evaluate performance of the proposed TNN search algorithms.
\end{abstract}

\section{Introduction}

With ubiquitous deployment of wireless networks and sky rocketing popularity of smart mobile devices, there is a strong demand in wireless data services. Among them, location-based services (LBSs), providing clients with the right information at the right place, stand out as a killer application because location information is critical to many applications, ranging from crisis management, public health, national security, to international commerce [11]. For example, Federal Communications Commission mandates wireless carriers to provide precise location information of E911 calls from wireless phones (see http://www.fcc.gov/911/enhanced). NextBus (http://www.nextbus.com) uses GPS to provide real-time arrival information of public transit, shuttles, and trains to specified stops with live updates. MSN Direct Service (http://www.msndirect.com) provides localized timely information such as local news, weather, and traffic information to its subscribers, via a continuous broadcast network using FM radio subcarrier frequencies.

An important functionality of LBSs is to answer location-based queries, e.g. range query and nearest neigh-

${ }^{*}$ Wang-Chien Lee and Ken C.K. Lee were supported in part by US National Science Foundation Grant IIS-0328881. bor search. In this paper, we study a new type of locationbased queries - transitive nearest neighbor (TNN) search. For a given query point $p$ and two datasets $\mathcal{S}$ and $\mathcal{R}$, TNN returns a pair of objects $(s, r) \in \mathcal{S} \times \mathcal{R}$, such that $\forall\left(s^{\prime}, r^{\prime}\right) \in \mathcal{S} \times \mathcal{R},(\operatorname{dis}(p, s)+\operatorname{dis}(s, r)) \leq\left(\operatorname{dis}\left(p, s^{\prime}\right)+\right.$ $\left.\operatorname{dis}\left(s^{\prime}, r^{\prime}\right)\right)$ where $\operatorname{dis}(p, s)$ represents the distance between two points $p$ and $s$. Each dataset in the query, corresponding to a particular type of spatial objects (e.g., restaurants, hotels, etc), is called a destination set. By specifying a query point (which usually is the current position of a client) and two destination sets in order, TNN finds a pair of data objects from the corresponding destination sets, which provides the shortest total distance to the query point.

Applications of TNN exist everywhere in our daily life. For example, Amy needs to drop off some clothes at a dry cleaner and get some flowers for a friend's birthday. TNN can help Amy to decide which dry cleaner and florist to go to in a single trip with the shortest distance. Bobby is planning for a date in Friday evening. A TNN search can help him to locate a gourmet restaurant and a nearby movie cinema not far away from city center. Even with such a broad application base, there is no existing study on TNN appeared in the literature. To the best knowledge of the authors, this is the first research on this new query.

On-demand data access and periodic data broadcast are two primary approaches for provisioning mobile services. In this paper, several alternative algorithms for TNN query processing have been proposed: Multiple-Nearest-Neighbor-Search method and Assistance-TNN-Search method for on-demand mobile services while Window-Based-TNN-Search method and Approximate-TNN-Search method for broadcast-based mobile services. Additionally, we develop a novel validation algorithm that allows the clients to verify whether TNN answers are still valid after the clients move to new positions so that unnecessary TNN query reevaluations for same answers are avoided. Finally, we conduct a comprehensive simulation to evaluate performance of our proposal.

The rest of this paper is organized as follows. Section 2 discusses the system model and related work. Details of the proposed algorithms for supporting TNN queries are de- 
scribed in Section 3. A comprehensive evaluation of our proposal is conducted in Section 4. Finally, this paper is concluded in Section 5.

\section{Preliminaries}

In this section, we first describe the system model, assumptions and constraints of mobile systems. Next, we review the R-tree index that our proposed algorithms are based on and the classical nearest neighbor (NN) search algorithm. Some other variants of NN search algorithm are also briefly discussed.

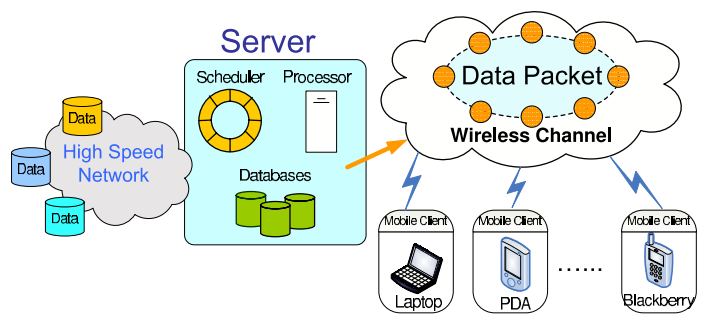

Figure 1. System Model for Wireless Data Services

\subsection{System Model}

A wireless data service in a mobile environment consists of three parts: 1) the communication mechanism; 2) the server; and 3) the mobile clients. Figure 1 shows a highlevel view of the system model. The wireless channel is the main communication mechanism between the clients and the server. We assume that the information transferred in a wireless channel is in the unit of page (or data packet shown in the figure). The server is interfaced with other data sources via high-speed networks and thus can be considered as a logical data source for all the mobile clients in the system. Thus, we assume that the server has a full knowledge of all data objects requested by the clients. A data object consists of a set of attributes and a content body. Among all attributes, location attribute is particularly important in the context of this paper. We assume the location attribute maintains a geospatial coordinate.

The server provides data services to the mobile clients via either on-demand access or periodic broadcast. The former represents the conventional client-server model where a mobile client submits a request, together with its current location, to the server via a dedicated point-to-point channel ${ }^{1}$. After processing the query, the server sends back the answer to the client via the point-to-point channel. On the other hand, periodic broadcast, a complement alternative to the on-demand approach, is particularly useful when an uplink channel is not available. A server periodically broadcasts data via a public channel. A mobile client continuously monitors the channel to retrieve the interested data objects in order to answer its queries locally. Different broadcast

\footnotetext{
${ }^{1}$ Current positioning technology (e.g., GPS) is available for clients to obtain their own positions.
}

programs based on query access patterns have been proposed [1]. In order to simplify our discussion, we assume a flat broadcast, i.e., each data object is broadcast only once in a broadcast cycle, the duration in which the whole dataset is broadcast once.

This study uses response time and search cost as the primary performance metrics. The former is the time elapsed between the moment when a query is issued to the moment when the query is answered. The latter represents the number of page accesses in order to finish a query. The ultimate objective is to answer a TNN query with short response time and low search cost. Since indexing techniques are commonly used to accelerate query evaluation, we propose several search algorithms based on R-tree index [4] for its popularity and well-acceptance.

The two mobile data access approaches are functionally different. In the on-demand mode, indexes are stored in memory and hard disks, while the index in the wireless broadcast mode is only available "on air" which is perceived as a linear stream of data pages flowing along the time axis. Consequently, the pointers in air index can only tell the upcoming broadcast time, which is relative to the current time, of the corresponding objects. A miss of a data object forces the client to wait until the object is rebroadcast in the next broadcast cycle, therefore prolonging the response time. As a result, the pre-set broadcast order of the index and objects decides the access order of the pages. On the other side, dynamic access order, which is supported in on-demand access mode, is no longer available for broadcast-based systems. An example to be discussed in the next section will further illustrate the difference of these two mobile data access approaches.

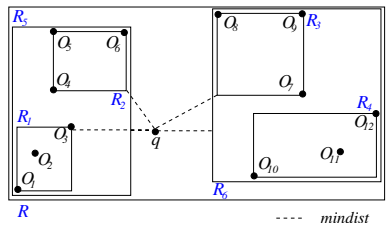

(a) Objects and MBR Structure

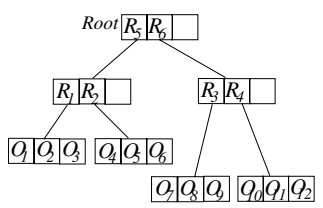

(b) R-tree Index
Figure 2. Nearest Neighbor Search on R-tree

\subsection{Related Work}

R-tree[4] is one of the most well-known spatial indices. It recursively groups objects into minimal bounding rectangles (MBRs) until the whole data space is fully covered by one MBR, i.e., the root of the tree. Figure 2 depicts 12 objects and the corresponding R-tree, with 3 as the fanout.

To perform NN search, a branch-and-bound approach is often employed to traverse the index. At each step, heuristics are applied to order branches to visit and the search space is continuously refined. Several R-tree-based NN search algorithms are proposed, and they mainly differ in the searching order and the heuristics used to prune the 
branches $[2,5,10]$. Take an NN query issued at point $q$ in Figure 2(a) as an example. Best-First (BF) search algorithm [5], the most efficient NN search algorithm, maintains a priority queue to keep all the candidate nodes, which are sorted based on mindist to the query point ${ }^{2}$.

This BF search algorithm determines the access order of the index nodes subject to the query issuing locations. If we apply this algorithm in the broadcast mode, the performance deteriorates significantly because of linear delivery properties of broadcast. For example, R-tree nodes are broadcast based on the left-first traversal order as depicted in Figure 3. The client will retrieve node $R_{2}$ before node $R_{1}$, while node $R_{1}$ is broadcasted prior to that of $R_{2}$. As a result, when the client wants to download node $R_{1}$ after accessing node $R_{2}$, node $R_{1}$ has already been broadcast and thus the client has to wait till the next time it is broadcast (as illustrated by the last arc in Figure 3). The response time is extended every time the access order differs from the broadcast order. Therefore, search algorithms developed for wireless broadcast systems have to cater for this linear access characteristics.

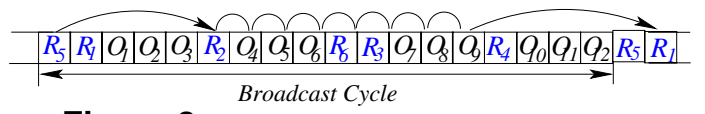

Figure 3. Linear Access in Broadcast Model

Due to the importance and popularity of NN problem, some variants have been well studied. Continuous nearest neighbor $(\mathrm{CNN})$ problem is to find the nearest neighbors to all the points along a query line segment [12], e.g. a mobile client continuously issuing a NN search while moving. Reverse nearest neighbor (RNN) query is to retrieve all point objects $p$ that are near neighbor to a query point, $q$ [7]. All nearest neighbor search is to find for each query point in a query data set, $\mathcal{Q}$, a nearest neighbor in object data set $\mathcal{P}$ [13]. Group nearest neighbor (GNN) query is to find an object, $o$, in a dataset that produces the smallest sum of distances from a set of $n$ query points, $q_{1}, q_{2}, \cdots q_{n}$ [9]. Formally, the distance metric is expressed as $\sum_{i}^{n} \operatorname{dist}\left(q_{i}, o\right)$. However, up to the authors' knowledge, no existing work has addressed the TNN search issue and this is the first work to introduce this TNN query.

\section{Answering TNN Queries}

In this section, we propose several search algorithms to answer TNN queries in on-demand and broadcast environments. The first two algorithms are for the on-demand environment in which queries are evaluated with aids of diskbased indexes, while the other two algorithms are based on the broadcast environment in which clients tune in the wireless broadcast to find required data. The following descriptions assume R-tree as the underlying index. However, the

\footnotetext{
${ }^{2}$ mindist $(R, p)$ returns the minimal possible distance between a query point $p$ and any point in an $\mathrm{MBB}, R$.
}

algorithms can be straightforwardly applied to other spatial index structures. We then address the answer validation issue; a novel algorithm is proposed to enable a client to detect the validity of returned answer as she moves to a new position that is different to the position where she issued the query. In order to simplify the discussion, we assume the issued TNN query is to retrieve a pair of objects $(s, r)$ from two datasets, $\mathcal{S}$ and $\mathcal{R}$. A running example is depicted in Figure 4, with $\mathcal{S}=\left\{s_{1}, s_{2}, s_{3}, s_{4}, s_{5}\right\}$ and $\mathcal{R}=\left\{r_{1}, r_{2}, r_{3}\right\}$. Table 1 summarizes the terminology used in our discussion.

\begin{tabular}{|l||l|}
\hline Notation & Description \\
\hline \hline $\operatorname{dis}(p, s)$ & Euclidean distance between points $p$ and $s$ \\
\hline$p . N N(\mathcal{S})$ & point $p$ 's nearest neighbor in set $\mathcal{S}$ \\
\hline $\operatorname{cir}(p, r)$ & a circle centered at point $p$ with $r$ as the radius \\
\hline$T N N(p)$ & the transitive nearest neighbors to query point $p$ \\
\hline$D_{T N N}(p)$ & $\begin{array}{l}\text { the distance between query point } p \text { and the corre- } \\
\text { sponding transitive nearest neighbors }\end{array}$ \\
\hline
\end{tabular}

Table 1. Terminology Definition

\subsection{On-Demand Access}

Without loss of generality, we assume that the server contains multiple datasets of heterogeneous types, such as restaurants, ATM, cinemas, shopping malls and so on, and that an R-tree index for each dataset is available.

A TNN query may involve any two arbitrary datasets. Such a combination of datasets is specified by a query in an ad hoc fashion. Multiple-NN-Search method is proposed to handle TNN query in such a dynamic case. In contrast, if the combination of datasets involved in queries is very common, Assistant-TNN-Search method can be adopted. Detailed descriptions are provided as follows.

Multiple-NN-Search Method As the name suggests, Multiple-NN-Search invokes more than one NN search arranged in a nested loop manner to determine TNN. The first NN search (in the outer loop) is to find the nearest object $s$, with respect to a query point $p$ in dataset $\mathcal{S}$. The second NN search (in the inner loop) is to search the nearest object $r$ to $s$ in dataset $\mathcal{R}$. A probe distance is introduced to prune the search space effectively. To illustrate, let us see an example.

Suppose a query is issued at a point $p$ as shown in Figure 4, Multiple-NN-Search method starts the processing by retrieving $p$ 's first $\mathrm{NN}$ object (i.e., $s_{1}$ ) in $\mathcal{S}$ and $s_{1}$ 's NN object in $\mathcal{R}$ (i.e., $r_{2}$ ). The probe distance $d$ is thereafter set to $\operatorname{dis}\left(p, s_{1}\right)+\operatorname{dis}\left(s_{1}, r_{2}\right)=5$. This probe distance $d$ is defined as the upper bound of $D_{T N N}(p)$. The $p$ 's second $\mathrm{NN}$ object in $\mathcal{S}$ is $s_{5}$ and its distance to $p$ (i.e., $\operatorname{dis}\left(p, s_{5}\right)=2.5$ ) is smaller than the current probe distance $d$. Therefore, the NN search continues. After $s_{5}$ 's NN object $r_{1}$ in $\mathcal{R}$ is accessed, the probe distance shrinks to $d=$ $\operatorname{dis}\left(p, s_{5}\right)+\operatorname{dis}\left(s_{5}, r_{1}\right)=3.5$. Then, $p$ 's third NN object (i.e., $\left.s_{4}\right)$ in $\mathcal{S}$ has no shorter distance to $p\left(\operatorname{dis}\left(p, s_{4}\right)=4\right)$ than the current probe distance (3.5) and hence the search 
can be safely terminated. The detailed pseudo code is ignored to save space.

Assistant-TNN-Search Method A particular TNN query may only cover a fixed pair of datasets. For example, queries asking for dating for Friday evening might be only interested in restaurants and then cinemas. If the datasets frequently involved in certain TNN queries are known in advance, data can be pre-processed to improve the search efficiency.

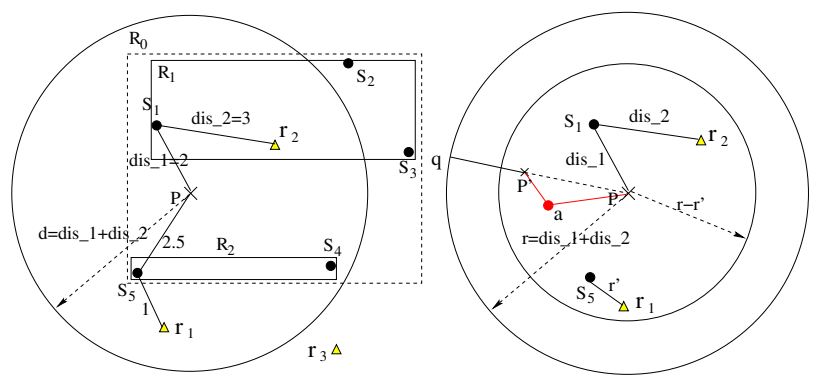

Figure 4. TNN Search Range

Figure 5. Valid Region

Due to the fact that the object $r \in \mathcal{R}$ in the answer set $T N N(p)=(s, r)$ must be the nearest neighbor to object $s \in \mathcal{S}$ in the dataset $\mathcal{R}$, i.e., $r=s . N N(\mathcal{R})$, all $s$ 's nearest neighbor in $\mathcal{R}$ can be determined in advance. In this case, an all-nearest-neighbor search algorithm can be adopted to find, for each object $s$ in the dataset $\mathcal{S}$, the corresponding nearest neighbor, $s . N N(\mathcal{R})$, in the dataset $\mathcal{R}$ [13]. We present an enhanced R-tree index structure such that TNN processing needs only scanning the index of $\mathcal{S}$ once. The structures of R-tree nodes are changed accordingly to capture the nearest neighbor information. For each leaf node, an additional tuple $[o b j, d i s]$ is augmented to each object $s$, with $o b j=s \cdot N N(\mathcal{R})$ and dis $=\operatorname{dis}(s, o b j)$. Similarly, each internal node maintains two parameters $d_{\text {min }}$ and $d_{\max }$, respectively, representing the minimal and maximal of the dis attributes of its descendant leaf nodes. For example, the modified $\mathrm{R}$-tree for the dataset $\mathcal{S}$, as shown in Figure 4, is depicted in Figure 6. Like the $\left[r_{2}, 3\right]$ in the leftmost leaf node means $r_{2}$ is the NN object to $s_{1}$ with a distance of 3. Similarly, a pair of numbers in the square brackets are $d_{\min }$ and $d_{\max }$. Given an R-tree, the detailed settings of $d i s, d_{\text {min }}$, and $d_{\text {max }}$ attributes at different nodes can be propagated from the leaf nodes up to the root node.

The search algorithm maintains a priority queue initialized with the root node of the index. All the nodes in queue are sorted in an increasing order of nodes' mindist* values $^{3}$. Nodes are removed from the queue if their mindist* are larger than the current probe distance whose initial value is infinity. When a dequeued node is visited, all its child nodes are inserted into the queue if it is an internal node.

\footnotetext{
${ }^{3}$ mindist ${ }^{*}$ of an entry $e$ is the summation of original mindist and e.d $d_{\text {min }}$.
}

If the dequeued node is a leaf node, all underlying objects and the probe distance are retrieved, and then the content of the queue is updated accordingly. The search is completed when the queue is empty. Figure 6 lists the search steps involved for the TNN query issued at the point $p$ in Figure 4.

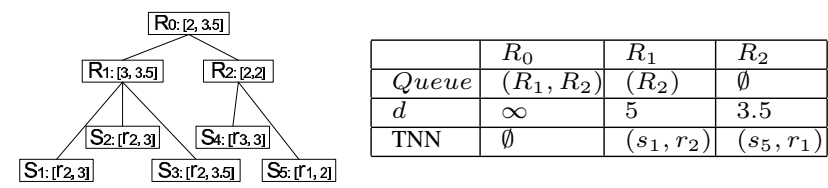

Figure 6. Assistant TNN Search Method

\subsection{Wireless Broadcast}

As discussed in the previous section, Multiple-NNSearch method is expected to perform reasonably well in the on-demand access mode. However, it incurs multiple scans of indexes which is a potential pitfall, especially when the object density of dataset $\mathcal{S}$ is much higher than that of $\mathcal{R}$ in this situation, such that the expected distance $\operatorname{dis}(s, r)$ is much larger than expected distance $\operatorname{dis}(p, s)$ and therefore it dominates the probe distance. As a result, a large number of NN objects from $\mathcal{S}$ have to be retrieved. This situation will become even worse in the broadcast environment where objects are broadcast according to a fixed order. Suppose an R-tree is broadcast $m$ times within one broadcast cycle and $n \mathrm{NN}$ queries are issued to finish one TNN, the average response time is $n / m$ broadcast cycles. In order to answer TNN queries with a stable and competitive response time in broadcast environments, two new search algorithms which incur a small and fixed number of index scans are proposed to answer TNN. Both algorithms map a TNN search into window queries but differ in the way to decide the size of corresponding windows.

Window-Based-TNN-Search Method This method issues only two NN searches, one to retrieve $s(=p . N N(\mathcal{S}))$ from dataset $\mathcal{S}$ and the other to retrieve $s$ 's NN object from dataset $\mathcal{R}$. Based on the detected $\mathrm{NN}$ objects, a search range which bounds the answer objects is decided. Thereafter, two window queries are issued to find all the candidate objects in both datasets. Finally, a refinement process is invoked to obtain the real answer. Before we present the detailed search algorithm, the following theorem is introduced.

Theorem 1. Given a query point $p$ and a pair of objects $(s, r) \in \mathcal{S} \times \mathcal{R}$, let $d=\operatorname{dis}(p, s)+\operatorname{dis}(s, r)$. If $s^{\prime} \notin$ $\operatorname{cir}(p, d)$ with $s^{\prime} \in \mathcal{S}$, it is guaranteed that $s^{\prime} \notin T N N(p)$. Similarly, if $r^{\prime} \notin \operatorname{cir}(p, d)$ with $r^{\prime} \in \mathcal{R}$, it is guaranteed that $r^{\prime} \notin T N N(p)$.

Proof: Suppose the object $T N N(p)=\left(s^{\prime \prime}, r^{\prime \prime}\right)$ and object $s^{\prime \prime}$ is not covered by the circle $\operatorname{cir}(p, d)$. Based on the definition of TNN, $d^{\prime \prime}=\operatorname{dis}\left(p, s^{\prime \prime}\right)+\operatorname{dis}\left(s^{\prime \prime}, r^{\prime \prime}\right)$ is minimized, i.e., $d^{\prime \prime} \leq d$. Therefore, $\operatorname{dis}\left(p, s^{\prime \prime}\right) \leq d^{\prime \prime} \leq d$. In other words, object $s^{\prime \prime}$ must lie inside the circle $\operatorname{cir}(p, d)$. 
Consequently, the assumption is not satisfied. Similarly, $r \in T N N(p)$ must lie inside $\operatorname{cir}(p, d)$. The proof is finished.

Theorem 1 provides a heuristic to prune the search space. As long as a pair of objects $(s, r)$ is retrieved, the search space can be significantly shrunk to a circle with radius set to $d(=\operatorname{dis}(p, s)+\operatorname{dis}(s, r))$. Since the value of $d$ has a direct impact on the search performance, the selection of the candidate pair is critical. Given the fact that only the location of the client, i.e., $p$, is available, the best way to minimize $d$ is to minimize $\operatorname{dis}(p, s)$, i.e., $s=p \cdot N N(\mathcal{S})$. Based on the detected object $s$, its nearest neighbor $r$ in the dataset $\mathcal{R}$ can be retrieved as well. In other words, $(s=p \cdot N N(S), r=s . N N(R))$ forms a candidate set. Thereafter, two window queries are issued at $p$ with radius $d=\operatorname{dis}(p, s)+\operatorname{dis}(s, r)$ to retrieve objects from datasets $\mathcal{S}$ and $\mathcal{R}$, respectively. Finally, a join algorithm can be adopted to find out the final answer.

Back to the running example shown in Figure 4, Window-Based-TNN-Search method first retrieves $p . N N(\mathcal{S})$ (i.e., $\left.s_{1}\right)$ and $s_{1} . N N(\mathcal{R})$ (i.e., $r_{2}$ ). Thereafter, the search radius $d$ is fixed and objects $\left(s_{1}, s_{4}, s_{5}\right)$ and $\left(r_{1}, r_{2}\right)$ are retrieved. The final answer $\left(s_{5}, r_{1}\right)$ can be easily detected based on distance calculation.

Approximated-TNN-Search Method The previous search algorithm needs to traverse an index four times (i.e., two for NN searches and two for window queries). We further reduce the number of traversals to two by introducing the Approximated-TNN-Search method. The basic idea is to decide the search range based on approximation, rather than using two NN queries. For a given dataset, the radius of a circle that encloses at least $k$ objects can be derived as Equation (1), which is query independent. As a result, a TNN query based on two given datasets, $\mathcal{S}$ and $\mathcal{R}$, has a fixed search range, i.e., $d=r_{1}(\mathcal{S})+r_{1}(\mathcal{R})$.

$$
r_{k}(\mathcal{S})=\ln (n) \times \sqrt{\frac{k}{(\pi \times n)}}, \text { where } n=|\mathcal{S}|
$$

Claim 1 Assume objects of dataset $\mathcal{S}$ are uniformly distributed in a unit square, i.e., $1 \times 1$. For any given $k$ $(k<<n)$ and a circle having a radius as obtained by Equation (1), $\lim _{k<<n} \operatorname{Pr}[X \leq k]=0$ where $X$ is the number of objects within that circle. The proof is ignored due to space limitation.

Therefore, with the increase of objects counts, the probability that the number of objects within the given circle is smaller than $k$ decreases exponentially and eventually converges to zero. Consequently, our claim holds. In the above proof, the objects are assumed to be uniformly distributed. However, when the objects' distribution is clustered or even skewed (i.e., non-uniformly distributed), Equation (1) still can provide some useful guidance. This has been verified using real data in the performance evaluation section.

\subsection{Answer Validation}

Mobile clients may continue to move right after they are issuing queries. In most cases there is a position change between the location a query is issued and that the result is received. Therefore, Answer validation becomes extremely important for location based queries in order to ensure the result accuracy. Answer validation for TNN query enables a client to check whether the received TNN answer set is still the right answer according to its current location. Existing approaches tackle this problem by providing clients a valid region along with returned answer, within which the returned answer is guaranteed to be correct $[14,15]$. However, these existing approaches only consider simple queries such as NN and window queries. The computation of a valid region for TNN search is more complicated than the ordinary ones. Rather than calculating the irregular valid region with high computation cost, we propose a novel algorithm to enable validation while computation cost is kept minimized. Before presenting the detailed algorithm, we first introduce the following theorem based on which the validation algorithm is developed.

Theorem 2. Given a TNN query for datasets $\mathcal{S}$ and $\mathcal{R}$ issued at $p, T N N(p)=(s, r)$. Let object pair $\left(s_{2}, r_{2}\right)$ be the pair such that $d=\left(\operatorname{dis}\left(p, s_{2}\right)+\operatorname{dis}\left(s_{2}, r_{2}\right)\right) \leq$ $\operatorname{dis}\left(p, s^{\prime}\right)+\operatorname{dis}\left(s^{\prime}, r^{\prime}\right), \forall\left(s^{\prime}, r^{\prime}\right)(\neq(s, r)) \in \mathcal{S} \times \mathcal{R}$. Suppose $p^{\prime} \in \operatorname{cir}(p, d)$, if $T N N\left(p^{\prime}\right) \neq(s, r), D_{T N N}\left(p^{\prime}\right)$ is bounded by the distance between $p^{\prime}$ and its nearest point along the circle $\operatorname{cir}(p, d)$.

Proof: Suppose there is a point $p^{\prime} \in \operatorname{cir}(p, d)$ and $T N N\left(p^{\prime}\right)=(a, b)$ with $(a, b) \neq(s, r)$. It is well known that the nearest point along the circle to a point $p^{\prime} \in$ $\operatorname{cir}(p, d)$ is the intersection between the circle and the line formed by points $p$ and $p^{\prime}$ (as denoted by point $q$ in Figure 5). If Theorem 2 is incorrect, $\operatorname{dis}\left(p^{\prime}, a\right)+\operatorname{dis}(a, b) \leq$ $\operatorname{dis}\left(p^{\prime}, q\right)$. Based on the triangle theorem, $\operatorname{dis}(p, a) \leq$ $\operatorname{dis}\left(p^{\prime}, a\right)+\operatorname{dis}\left(p^{\prime}, p\right)$. As a result, $\operatorname{dis}(p, a)+\operatorname{dis}(a, b)<$ $\operatorname{dis}\left(p^{\prime}, a\right)+\operatorname{dis}\left(p^{\prime}, p\right)+\operatorname{dis}(a, b)<\operatorname{dis}\left(p^{\prime}, p\right)+\operatorname{dis}\left(p^{\prime}, q\right)=$ $d$.

As we have stated in Theorem 2, $d \leq \operatorname{dis}\left(p, s^{\prime}\right)+$ $\operatorname{dis}\left(s^{\prime}, r^{\prime}\right), \forall\left(s^{\prime}, r^{\prime}\right)(\neq(s, r)) \in \mathcal{S} \times \mathcal{R}$. Therefore, the assumption is not satisfied and the proof is completed.

Based on Theorem 2, the validation process at client side can be proceeded accordingly. Given the answer set to a TNN query and corresponding $d$ as defined in Theorem 2, clients can easily check whether the answer is still the right answer to her current location. This validation algorithm needs to take only two Euclidean distance calculations. The accuracy of the validation algorithm can be easily derived based on Theorem 2 and the detailed proof is omitted for space saving. Now the only question left is how to obtain the value of $d$. Although there are many possible approaches to determine the value of $d$, we adopt a simple one that only incurs the least overhead in terms of number of 
extra queries to process. We simply find $2 \mathrm{TNN}$ instead of TNN (please refer to the next subsection for detailed definition and search algorithm of $k \mathrm{TNN}$ ).

\subsection{Discussion}

The proposed search algorithms can be extended to support variants of TNN search. Due to space limitation, we discuss a few representative ones and their corresponding extensions. The first variant is $k$ TNN queries, whose definition is summarized as follows. For a given query point $p$, it returns $k$ pairs of objects Res $=\left\{\left(s_{i}, r_{i}\right) \mid(i \in[1, k]),\left(s_{i}, r_{i}\right) \in\right.$ $\mathcal{S} \times \mathcal{R} s . t . \forall\left(s^{\prime}, r^{\prime}\right) \in(\mathcal{S} \times \mathcal{R}-\operatorname{Res}), \quad\left(\operatorname{dis}\left(p, s^{\prime}\right)+\right.$ $\left.\left.\operatorname{dis}\left(s^{\prime}, r^{\prime}\right)\right) \geq M A X\left(\operatorname{dis}\left(p, s_{i}\right)+\operatorname{dis}\left(s_{i}, r_{i}\right)\right)\right\}$. For Multiple-NN-Search method, for each returned NN object from the dataset $\mathcal{S}$, its $k \mathrm{NN}$ objects from dataset $\mathcal{R}$ are returned and a queue is maintained to keep up to $k$ pair of objects with smaller total distance to the query point than other detected pairs. The probe distance is thereafter defined as the maximal distance among $k$ object pairs in the queue. When a returned NN object from the dataset $\mathcal{S}$ has a longer distance to query point $p$ than the probe distance, the query is finished. For Assistant-TNN-Search method, the probe distance $d$ is set when there are $k$ pairs of objects retrieved. Therefore, instead of keeping one pair of candidate objects, a queue of $k$ pairs has to be maintained.

For Window-Based-TNN-Search method in the wireless broadcast systems, $k \mathrm{NN}$ objects (denoted by $\left\{s_{i} \mid i \in\right.$ $[1, k]\}$ ) to query point $p$ in dataset $\mathcal{S}$, together with the corresponding NN objects $r_{i}$ to $s_{i}$ in dataset $\mathcal{R}$ are retrieved, by calling the $k \mathrm{NN}$ search algorithm [10]. Each pair of objects $\left(s_{i}, r_{i}\right)$ bounds a certain range decided by $d_{i}=\operatorname{dis}\left(p, s_{i}\right)+\operatorname{dis}\left(s_{i}, r_{i}\right)$ and the maximal $d_{i}$ is set as the search radius $d$. The circle $\operatorname{cir}(p, d)$ is guaranteed to bound at least $k$ pairs of objects to ensure the accuracy.

TNN search based on multiple datasets is another variant. Suppose there are three datasets $(\mathcal{S}, \mathcal{R}$, and $\mathcal{O})$ involved, the returned result $(s, r, o) \in \mathcal{S} \times \mathcal{R} \times \mathcal{O}$ provides the smallest distance for a given query $p$, i.e., $\operatorname{dis}(p, s)+$ $\operatorname{dis}(s, r)+\operatorname{dis}(r, o) \leq \operatorname{dis}\left(p, s^{\prime}\right)+\operatorname{dis}\left(s^{\prime}, r^{\prime}\right)+\operatorname{dis}\left(r^{\prime}, o^{\prime}\right)$, $\forall\left(s^{\prime}, r^{\prime}, o^{\prime}\right) \in \mathcal{S} \times \mathcal{R} \times \mathcal{O}$. We can even assume the visiting order of objects from datasets $\mathcal{S}, \mathcal{R}$ is irrelevant. Back to the example we list in Section 1, Amy can either go to the dry cleaner first or go to the florist first as long as the travelling distance is minimized. Furthermore, we can consider the travel back to the source as well. Under this assumption, we target at a complete travel route, starting from a given source point, visiting several specific destinations, and then coming back to the source point or a given final destination. We leave the development of search algorithms in our future work.

\section{Performance Evaluation}

This section evaluates the performance of the proposed TNN search algorithms in the mobile environment, including both on-demand access scenario and wireless broadcast scenario. Both synthetic and real datasets are used in the evaluation. For the synthetic datasets $\operatorname{UNIF}(N), N$ objects in a square unit space are uniformly generated. For real datasets, $C I T Y$ contains around 6,000 cities and villages of Greece and POST contains over 120, 000 post offices in the northeast of the United States. Both datasets are extracted from the point dataset available in [3], and they are normalized to the same square unit space as UNIF datasets. The system parameters for the experiments are set as in Table 2. Without loss of generality, a uniform access pattern is assumed and the presented results are the average performance of 100, 000 randomly issued queries.

\begin{tabular}{|l|l|}
\hline Parameter & Setting \\
\hline \hline size of an index pointer & 2 bytes \\
size of a coordinate & 4 bytes \\
size of a data content & $1 k$ bytes \\
page capacity & $64-2 k$ bytes \\
\hline
\end{tabular}

Table 2. System Parameter Settings

R-tree is selected as the index structure to support TNN search. As the locations of data objects are known a priori, the STR packing scheme is employed to build R-tree in order to achieve the best performance [8].

Since R-tree algorithms typically rely on backtracking to traverse an index which is shown to be inefficient in the broadcast based systems, the proposed algorithms map the TNN search into window queries which do not incur backtracking are expected to perform better. However, for the NN search involved by the window-based TNN search method, the nodes are accessed based on their broadcast order.

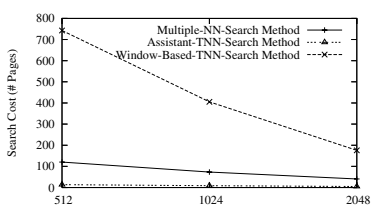

(a) $\mathcal{R}=\mathrm{UNIF}: \stackrel{\stackrel{\text { Page Capaciy (Byyes) }}{=}}{=} 100,000$

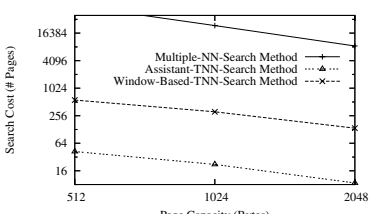

(b) $\mathcal{R}=\operatorname{CITY}: \stackrel{\text { Page Capaciy (Byes) }}{=} 5,848$
Figure 7. Search Cost vs. Page Capacity $(\mathcal{S}=$ POST: $N=103,630)$

\subsection{On-Demand Access}

The first experiment evaluates the proposed TNN search algorithms applicable to applied R-tree on disk in ondemand access environments. Although Window-BasedTNN-Search method is designed for wireless broadcast system, it can also be applied in on-demand access. We include it in this experiment for comparison. We measure the response time, as well as the average search cost, of evaluating TNN queries on the server. The size of the page is 
varied from $0.5 \mathrm{k}$, to $1 \mathrm{k}$, and to $2 \mathrm{k}$ bytes. Figure 7 shows the search cost in terms of number of page accesses as a function of page capacity. It is obvious that Assistant-TNNSearch method has a much better performance with the embedded nearest neighbor information.

The second interesting observation is that the performance of Multiple-NN-Search method is highly dependent on the size and objects distribution of the two involved datasets. As we mentioned before, the algorithm is terminated only when the distance between the query point $p$ and its $i$ th NN object of dataset $\mathcal{S}$ is longer than the detected total distance. When the dataset $\mathcal{R}$ has a much smaller size compared to $\mathcal{S}$, the distance $\operatorname{dis}(p, s)$ is only responsible for a small portion of the potential candidates. As a result, a large number of NN searches have to be issued. For example, when CITY is employed as the dataset $\mathcal{R}$, on average 691 objects from dataset $\mathcal{S}$ are retrieved when the page capacity is 512 bytes. Under this situation, Window-BasedTNN-Search algorithm has a much stable performance. On the other hand, when a uniform dataset with a similar number of objects as $\mathcal{S}$ is adopted, multiple-NN-search produces a comparable performance as Assistant-TNN-Search algorithm. This is because $\mathcal{S}$ is skewed while $\mathcal{R}$ is uniform. Therefore, the distance $\operatorname{dis}(p, s)$ has a bigger impact on the total distance and only a small number of NN searches are involved.

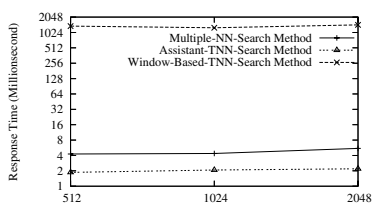

(a) $\mathcal{R}=\mathrm{UNIF}: \stackrel{\text { Pagec (capaiy (Byyes) }}{=} 100,000$

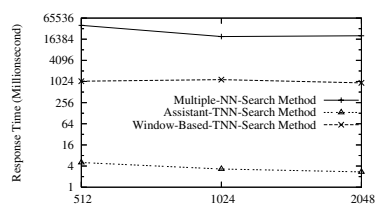

(b) $\mathcal{R}=\operatorname{CITY}: \stackrel{\text { Page Capaciy (Byyes) }}{=} 5,848$
Figure 8. Response Time vs. Page Capacity $(\mathcal{S}=$ POST: $N=103,630)$

The response time measuring the duration from a query is issued till the answer is returned. It consists of the time to transmit the query to the server, the time to evaluate the query at the server, and the time to return the answer to clients. Given the dedicated uplink/downlink channels, the time to upload a query and to download the answer is a constant. In this experiment, we only evaluate the average response time of processing TNN query at the server. Obviously Assistant-TNN-Search method is the most efficient owing to the pre-processing. The performance of Multiple-NN-Search and Window-Based-TNNSearch methods is again affected by the distribution and size of the involved datasets.

\subsection{Wireless Broadcast}

The second experiment evaluates the search algorithms in the wireless data broadcast environment where the index placement in the wireless channel usually has a direct impact on the response time. We assume the $(1, m)$ interleav- ing technique [6] is used to multiplex the index and data on the channel. It combines two datasets together and partitions the union into $m$ equal parts. The two corresponding $\mathrm{R}$-trees are broadcast as a unit before each of those $m$ parts. Please note that service providers might allocate multiple channels for each dataset. However, the placement issue is beyond the focus of this paper.

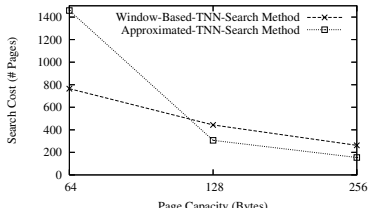

(a) $\mathcal{R}=\mathrm{UNIF}: \stackrel{\text { Page Capacity (Bytes) }}{=} 20,000$

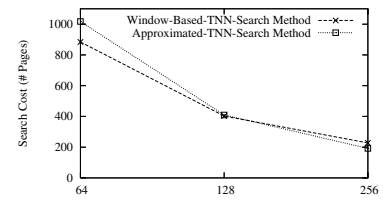

(b) $\mathcal{R}=\operatorname{CITY}: \stackrel{\text { Page Capacity (Bytes) }}{=} 5,848$

Figure 9. Search Cost vs. Page Capacity ( $\mathcal{S}=\mathrm{UNIF}: N=$ 10,000)

R-tree is broadcast in the depth-first order because breath-first broadcast requires a client to maintain a queue during query processing to keep track of the distance information between the query point and all the nodes in the same level in order to prune the unnecessary branches. The queue requires a large memory space, which may not be available to the mobile devices. Compared to the ondemand access mode, the datasets, as well as the page capacity, used in this experiment are much smaller due to the fact that broadcast service usually targets at a closed dataset which is relatively smaller with the page capacity varied from 128 bytes to 256 bytes.

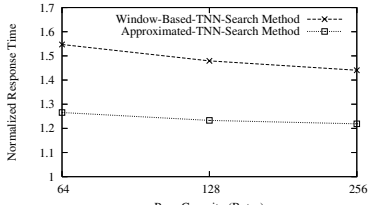

(a) $\mathcal{R}=\mathrm{UNIF}: \stackrel{\text { Page Capacity (Byyes) }}{=} 20,000$

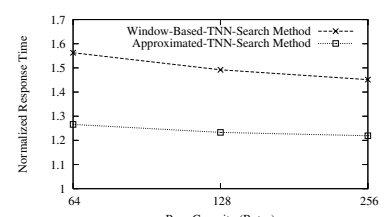

(b) $\mathcal{R}=\operatorname{CITY}: \stackrel{\text { Page Capaity (Byyes) }}{=} 5,848$
Figure 10. Normalized Response Time vs. Page Capacity $(\mathcal{S}=$ UNIF: $N=10,000)$

Figure 9 shows the search cost under different page capacities. The overall performances of those two methods are quite similar, since they both conduct window searches to answer TNN queries. Please note the approximation approach successfully predicts the search range for UNIF dataset, while it fails to answer around $10 \%$ of the issued queries when $\mathcal{R}=\mathrm{CITY}$. Therefore, its success rate on average is quite high. Furthermore, its failure only returns an empty set but not any wrong answer. Therefore, a client, when receiving an empty answer set, can either adopt window-based approach or slightly enlarge the search range.

On the other hand, approximation approach demonstrates a much better response time performance, as shown in Figure 10. We take an estimated optimal response time, i.e., a half of the broadcast cycle which consists of only data objects, as the baseline for comparison. For clarity of presentation, the experimental results are normalized based on 
this baseline response time. On average, approximation approach only prolongs the optimal response time by $24 \%$ and it only incurs $83 \%$ response time of the window-based approach. This is because the approximation approach saves two scans of the index.

\subsection{Answer Validation}

The third experiment evaluates the answer validation scheme. In the mobile environment, most of the clients keep moving even when they are issuing queries. Without answer validation, a client cannot validate whether the returned answer is still valid at its current location, which is very likely to be different from the location where the query is answered. The answer validation scheme proposed in this paper adopts a novel approach to enable an efficient validation checking. We evaluate the performance in terms of the allowed time difference between query issuing and answer receiving, within which the returned answer has the possibility to be the right answer. Since the moving velocity has a direct impact on the performance, the performance is showed as a function of the velocity. Without loss of generality, the velocity is set based on the side length of the service area. The presented result is the minimal time requested for a client to move across the returned circle which represents the allowed validity time in the worst case.

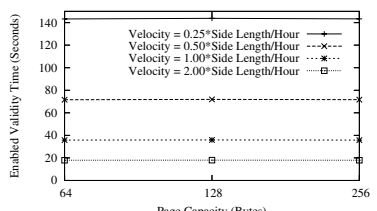

(a) $\mathcal{R}=\mathrm{UNIF}: \stackrel{\text { Page Capacily (Bytes) }}{=} 20,000$

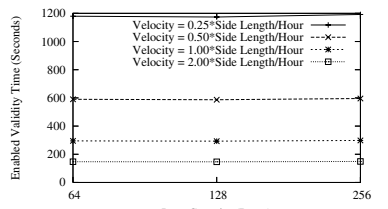

(b) $\mathcal{R}=\operatorname{CITY}: \stackrel{\text { Page Capacity (Byes) }}{=} 5,848$
Figure 11. Validity Time vs. Page Capacity $(\mathcal{S}=\mathrm{UNIF}$ : $N=10,000)$

Without answer validation, the client has to reissue the query if the answer is received at a location different from the query issuing point. It is obvious that the validation scheme does improve the efficiency of the system, saving lots of unnecessary re-issued queries. On the other hand, it serves as the guidance of the service planning. With the knowledge of the allowed time difference, the detailed system configuration, in terms of the computation ability of the server, the size of the cache, the allocated bandwidth, and other parameters, can be set accordingly to make sure that most clients can receive the answers to their TNN queries within the validity time.

\section{Conclusion}

With the advent of wireless networks and the popularity of mobile devices on the rise, the pervasive computing era arrive. Location-based services, which deliver data to clients based on not only the interest of clients but also the current location of clients, is a killer application for pervasive computing. In this paper, we study a new locationbased query, transitive nearest neighbor (TNN) search, in on-demand access or wireless broadcast environments.

We propose several algorithms for TNN searches and answer validation which is a necessity due to location changes of mobile clients. A comprehensive simulation has been conducted to evaluate the performance of proposed algorithms.

This paper only represents our first step towards the TNN queries in the mobile environments. As mentioned in Section 3.4, there are several interesting variants of TNN queries. We will continue the study in this direction and work out new solutions.

\section{References}

[1] S. Acharya, R. Alonso, M. Franklin, and S. Zdonik. Broadcast disks: Data management for asymmetric communications environments. In SIGMOD 1995, pages 199-210.

[2] K. L. Cheung and W.-C. Fu. Enhanced nearest neighbour search on the r-tree. SIGMOD Record, 27(3):16-21, 1998.

[3] Spatial Datasets. Website at http://dias.cti.gr/ ytheod/ research/datasets/ spatial.html.

[4] A. Guttman. R-trees: A dynamic index structure for spatial searching. In SIGMOD 1984, pages 47-54.

[5] Gí. R. Hjaltason and H. Samet. Distance browsing in spatial databases. TODS, 24(2):265-318, 1999.

[6] T. Imielinski, S. Viswanathan, and B. R. Badrinath. Data on air - organization and access. TKDE, 9(3), May-June 1997.

[7] F. Korn and S. Muthukrishnan. Influence sets based on reverse nearest neighbor queries. In SIGMOD 2000.

[8] S. T. Leutenegger, J. M. Edgington, and M. A. Lopez. Str: A simple and efficient algorithm for r-tree packing. In ICDE 1997, pages 497-506.

[9] D. Papadias, Q. Shen, Y. Tao, and K. Mouratidis. Group Nearest Neighbor Queries. In ICDE 2004, pages 301-312.

[10] N. Roussopoulos, S. Kelley, and F. Vincent. Nearest neighbor queries. In SIGMOD 1995, pages 71-79.

[11] Computer Science and Telecommunications Board. IT Roadmap to a Geospatial Future. The National Academies Press, 2003.

[12] A. P. Sistla, O. Wolfson, S. Chamberlain, and S. Dao. Modeling and querying moving objects. In ICDE 1997, pages 422-432.

[13] J. Zhang, N. Mamoulis, D. Papadias, and Y. Tao. AllNearest-Neighbors Queries in Spatial Databases. In SSDBM 2004, pages 297-306.

[14] J. Zhang, M. Zhu, D. Papadias, Y. Tao, and D. Lee. Locationbased spatial queries. In SIGMOD 2003.

[15] B. Zheng and D. L. Lee. Semantic caching in locationdependent query processing. In SSTD 2001, pages 97-116. 